\title{
EULERIAN DYNAMICS WITH A COMMUTATOR FORCING III. FRACTIONAL DIFFUSION OF ORDER $0<\alpha<1$
}

\author{
ROMAN SHVYDKOY AND EITAN TADMOR
}

To Edriss Titi with friendship and admiration

\begin{abstract}
We continue our study of hydrodynamic models of self-organized evolution of agents with singular interaction kernel $\phi(x)=|x|^{-(1+\alpha)}$. Following our works $[14,15]$ which focused on the range $1 \leqslant \alpha<2$, and Do et. al. [5] which covered the range $0<\alpha<1$, in this paper we revisit the latter case and give a short(-er) proof of global in time existence of smooth solutions, together with a full description of their long time dynamics. Specifically, we prove that starting from any initial condition in $\left(\rho_{0}, u_{0}\right) \in H^{2+\alpha} \times H^{3}$, the solution approaches exponentially fast to a flocking state solution consisting of a wave $\bar{\rho}=\rho_{\infty}(x-t \bar{u})$ ) traveling with a constant velocity determined by the conserved average velocity $\bar{u}$. The convergence is accompanied by exponential decay of all higher order derivatives of $u$.
\end{abstract}

\section{Contents}

1. Introduction and statement of main results. 1

2. Preliminary a priori bounds 4

3. Proof of the main result 5

3.1. Existence of global smooth solutions 5

3.2. Main theorem - step 1: exponential decay towards a flocking state 7

3.3. Main theorem - step 2: decay of higher derivatives 9

$\begin{array}{ll}\text { References } & 12\end{array}$

\section{IntRoduCtion AND STATEMENT OF MAIN RESUlts.}

We continue our study of one-dimensional Eulerian dynamics driven by forcing with a commutator structure initiated in [14, 15]:

$$
\left\{\begin{aligned}
\rho_{t}+(\rho u)_{x} & =0 \\
u_{t}+u u_{x} & =\mathcal{T}(\rho, u) .
\end{aligned}\right.
$$

Date: August 12, 2021.

1991 Mathematics Subject Classification. 92D25, 35Q35, 76N10.

Key words and phrases. flocking, alignment, fractional dissipation, Cucker-Smale, Motsch-Tadmor.

Acknowledgment. Research was supported in part by NSF grants DMS16-13911, RNMS11-07444 (KINet) and ONR grant N00014-1512094 (ET) and by NSF grant DMS 1515705 and the College of LAS, University of Illinois at Chicago (RS). Both authors thank the Institute for Theoretical Studies (ITS) at ETH-Zurich for the hospitality. 
The forcing $\mathcal{T}(\rho, u)$ takes the form $\mathcal{T}(\rho, u)=\left[\mathcal{L}_{\phi}, u\right](\rho):=\mathcal{L}_{\phi}(\rho u)-\mathcal{L}_{\phi}(\rho) u$, which involves the density $\rho$, the velocity $u$, and a convolution kernel $\phi$,

$$
\mathcal{L}_{\phi}(f):=\int_{\mathbb{R}} \phi(|x-y|)(f(y)-f(x)) \mathrm{d} y .
$$

The system arises as the macroscopic description for large-crowd dynamics of $N \gg 1$ "agents" driven by binary interactions through velocity alignment, [4],

$$
\left\{\begin{array}{l}
\dot{x}_{i}=v_{i}, \\
\dot{v}_{i}=\frac{1}{N} \sum_{j=1}^{N} \phi\left(\left|x_{i}-x_{j}\right|\right)\left(v_{j}-v_{i}\right), \quad\left(x_{i}, v_{i}\right) \in \Omega \times \mathbb{R}, \quad i=1,2, \ldots, N .
\end{array}\right.
$$

The kernel $\phi$ regulates the binary interactions among agents in $\Omega$. In the original setting of [4], $\phi$ is assumed positive, bounded influence function. Many aspects of the formal passage from (1.3) to (1.1) are discussed in e.g., [7, 1] and references therein; consult $[11,12]$ for singular $\phi$ 's. The important dynamical feature of the model is encoded in its long time behavior describing a flocking phenomenon, in which the crowd of agents congregates within a finite diameter $D(t)=\sup _{i, j}\left|x_{i}(t)-x_{j}(t)\right|<D_{\infty}<\infty$, while aligning their velocities, $\sup _{i, j} \mid v_{i}(t)-v_{j}(t) \stackrel{t \rightarrow \infty}{\longrightarrow} 0$, thus approaching the conserved average velocity, $v_{j}(t) \stackrel{t \rightarrow \infty}{\longrightarrow} \frac{1}{N} \sum_{k} v_{k}(0)$. Starting with the seminal work of Cucker and Smale paper [4] and the follow-up works $[7,6,10,17,2,15]$ and reference therein, it has become clear that in order to achieve unconditional flocking in either the agent-based or the macroscopic descriptions (1.3),(1.1), the system has to involve long range interactions so that $\int \phi(r) \mathrm{d} r=\infty$. The drawback of such an assumption in the context of Cucker-Smale model (1.3) is that each agent has to "count" all its $(N-1)$ neighbors, close and far with equal footing. To remove this deficiency, Motsch and Tadmor introduced in [9] an adaptive averaging protocol in which each neighboring agents is counted by its relative influence. Thus, the normalization pre-factor $1 / N$ on the right of (1.3) is replaced by $1 / \sum_{j} \phi\left(\left|x_{i}-x_{j}\right|\right)$, leading to the Eulerian dynamics (1.1) with non-symmetric forcing $\mathcal{T}(\rho, u)=\left[\mathcal{L}_{\phi}, u\right](\rho) /(\phi * \rho)$. The model is argued in [9] as more realistic in both - close to and away from equilibrium regimes, but its lack of symmetry is less amenable to the spectral analysis available in the symmetric Cucker-Smale model (1.3). An alternative approach was proposed by us in [14, 15], where nearby interactions are highlighted by the singularity of the interaction kernel at the origin, thus "adapting" different footing of neighboring agents by placing substantially smaller weights to those agents at far away distances relative to those nearby. A natural example is given by the power-law singularities $|x|^{-(1+\alpha)}, \alpha>0$. We consider the system (1.1) on the torus $\mathbb{T}$ with the $2 \pi$-periodized version of such kernels ${ }^{1}$

$$
\phi_{\alpha}(x):=\sum_{k \in \mathbb{Z}} \frac{1}{|x+2 \pi k|^{1+\alpha}}, \quad 0<\alpha<2 .
$$

which preserve the essential long range but less dominant interactions. In this case the operator $\mathcal{L}_{\alpha}=\mathcal{L}_{\phi_{\alpha}}$ becomes the (negative of) classical fractional Laplacian, $\mathcal{L}_{\alpha}=-\Lambda^{\alpha}$, which we denote

$$
\Lambda^{\alpha} u(x)=\int_{\mathbb{R}}(u(x)-u(x+z)) \frac{\mathrm{d} z}{|z|^{1+\alpha}}=\int_{\mathbb{T}}(u(x)-u(x+z)) \phi_{\alpha}(z) \mathrm{d} z, \quad \Lambda^{\alpha}=(-\Delta)^{\alpha / 2} .
$$

\footnotetext{
${ }^{1}$ We can in fact have an arbitrarily large period.
} 
Here and below we assume that $u(\cdot, t)_{\mid \mathbb{T}}$ and likewise, $\rho(\cdot, t)_{\mid \mathbb{T}}$, are extended periodically onto the line $\mathbb{R}$. The commutator forcing on the right hand side of the momentum equation in (1.1) then becomes a fractional elliptic operator:

$$
\mathcal{T}(\rho, u)=-\left[\Lambda^{\alpha}, u\right](\rho)(x)=\int_{\mathbb{R}} \rho(x+z)(u(x+z)-u(x)) \frac{\mathrm{d} z}{|z|^{1+\alpha}}, \quad 0<\alpha<2,
$$

with the density controlling uniform ellipticity. Written in this form, system (1.1) resembles the fractional Burgers equation with non-local non-homogeneous dissipation.

In [14] we proved global existence of smooth solutions of $(1.1),(1.3)_{\alpha}$ in the range $1 \leqslant \alpha \leqslant$ 2 , with focus on the most difficult critical case $\alpha=1$. To this end we utilized refined tools from regularity theory of fractional parabolic equations to verify a Beale-Kato-Majda (BKM) type continuation criterion which guarantees that the solution can be extended beyond $T$ provided $\int_{0}^{T}\left|u_{x}(\cdot, t)\right|_{\infty} \mathrm{d} t<\infty$. Building upon the technique developed in [14], in [15] we proved that all regular solutions converge exponentially fast to a so called flocking state, consisting of a traveling wave, $\bar{\rho}(x, t)=\rho_{\infty}(x-t \bar{u})$, with a fixed speed $\bar{u}$,

$$
|u(\cdot, t)-\bar{u}|_{X}+|\rho(\cdot, t)-\bar{\rho}(\cdot, t)|_{Y} \stackrel{t \rightarrow \infty}{\longrightarrow} 0, \quad \bar{u}:=\frac{\mathcal{P}_{0}}{\mathcal{M}_{0}} .
$$

Here the average velocity, $\bar{u}$, is dictated by the conserved mass and momentum,

$$
\mathcal{M}_{0}=\int_{\mathbb{T}} \rho_{0}(x) \mathrm{d} x, \quad \mathcal{P}_{0}=\int_{\mathbb{T}}\left(\rho_{0} u_{0}\right)(x) \mathrm{d} x .
$$

Parallel to the release of $[14,15]$, Do et.al. in [5] treated the case $0<\alpha<1$, where they proved global existence result with fast alignment of velocities. Although on the face of it, the system for that $\alpha$-range seems supercritical, one can employ the known conservation law for $e=u_{x}-\Lambda^{\alpha} \rho$ to conclude a priori uniform $C^{1-\alpha}$ Hölder regularity of the velocity, so that the equation (1.1), (1.3) $\alpha$ is kept critical in the range $0<\alpha<1$. In [5], the authors use construction of a modulus of continuity, the celebrated method implemented in treating many critical equations such as Burgers and, most notably, critical SQG equation by Kiselev et. al. [8], in order to verify a Beale-Kato-Majda type criterion $\int_{0}^{T}\left|\rho_{x}(\cdot, t)\right|_{\infty}^{2} \mathrm{~d} t<\infty$, to guarantee continuation of the solution beyond $T$.

In this present paper we revisit the parameter range $0<\alpha<1$ using the fractional parabolic technique developed in our earlier works for the range $1 \leqslant \alpha<2$. As in [15], our methodology will be to extract quantitative enhancement estimates for the dissipation term, using an adaptation of the non-linear maximum principle as in Constantin and Vicol's proof for the critical SQG, [3], that yields global existence and, moreover, allows us to completely describe the long time behavior — exponential convergence towards a flocking state. The main result summarized in the following theorem covers the global regularity and flocking behavior for singular kernels in the unified range $0<\alpha<2$. The $(1 \leqslant \alpha<2)$-part of the theorem was covered already in [15, Theorem 1.3], quoted in (1.4) with $(X, Y)=\left(H^{3}, H^{2+\alpha}\right)$. The $(0<\alpha<1)$-part of the theorem is new.

Theorem 1.1 (Flocking for singular kernels of fractional order $\alpha \in(0,2)$ ). Consider the system (1.1),(1.3) $\alpha$ with singular kernel $\phi_{\alpha}(x)=|x|^{-(1+\alpha)}, 0<\alpha<2$, on the periodic torus $\mathbb{T}$, subject to initial conditions $\left(\rho_{0}, u_{0}\right) \in H^{2+\alpha} \times H^{3}$ away from the vacuum. Then it admits a unique global solution $(\rho, u) \in L^{\infty}\left([0, \infty) ; H^{2+\alpha} \times H^{3}\right)$. Moreover, the solution converges 
exponentially fast to a flocking state $\bar{\rho}=\rho_{\infty}(x-t \bar{u}) \in H^{2+\alpha}$ traveling with a finite speed $\bar{u}$, so that for any $s<2+\alpha$ there exists $C=C_{s}, \delta=\delta_{s}$ with

$$
|u(t)-\bar{u}|_{H^{3}}+|\rho(t)-\bar{\rho}(t)|_{H^{s}} \leqslant C e^{-\delta t}, \quad t>0, \quad \bar{u}:=\frac{\mathcal{P}_{0}}{\mathcal{M}_{0}} .
$$

We recall that the global existence part for $0<\alpha<1$ was first derived in Do et. al. [5]. Our alternative proof is along the lines of - and in fact simpler to handle than, the borderline case $\alpha=1$ in [14]. The result is a consequence of Lemma 3.1 below, which gives a direct control on BKM continuation criteria $\left|\rho_{x}(\cdot, t)\right|_{\infty}$, and consequently on $\left|u_{x}(\cdot, t)\right|_{\infty}$, uniformly in time. Most of our work is then devoted for obtaining quantitative bounds on long time behavior of the slopes and higher order derivatives of the solution in the $(0<\alpha<1)$-part of the theorem.

\section{PRELIMINARY A PRIORI BOUNDS}

We start by listing several structural features of the system (1.1),(1.3) $\alpha$ and some preliminary a priori bounds of its solutions. We refer to [5, 14, 15] for details.

- (Control of higher order regularity). The starting point is the conservation law for a new quantity :

$$
e_{t}+(u e)_{x}=0, \quad e:=u_{x}-\Lambda^{\alpha} \rho .
$$

Paired with the mass equation we find that the ratio $e / \rho$ satisfies the transport equation

$$
\frac{\mathrm{D}}{\mathrm{D} t}(e / \rho):=\left(\partial_{t}+u \partial_{x}\right)(e / \rho)=0 .
$$

Hence, starting from sufficiently smooth initial condition with $\rho_{0}$ away from vacuum, this gives a priori pointwise bound

$$
|e(x, t)| \lesssim \rho(x, t) .
$$

This argument can be bootstrapped to higher orders [14, Sec. 2]: the next order quantity $Q=(e / \rho)_{x} / \rho$ is transported

$$
\left(\partial_{t}+u \partial_{x}\right) Q=0, \quad Q:=(e / \rho)_{x} / \rho
$$

hence solving for $e^{\prime}(\cdot, t)$ we obtain the a priori pointwise bound

$$
\left|e^{\prime}(x, t)\right| \lesssim\left|\rho^{\prime}(x, t)\right|+\rho(x, t) .
$$

This can be iterated to any order yielding the high-order bounds

$$
\left|e^{(k)}(x, t)\right| \lesssim\left|\rho^{(k)}(x, t)\right|+\ldots+\rho(x, t), \quad k=0.1 .2 ., \ldots
$$

As observed in [14], the smallest order $L^{2}$-based regularity class for which (2.4) can be understood classically, and hence (2.3) holds at every point is the class $u \in H^{3}$, and (2.3) is the lowest order law among (2.6) which allows to close energy estimates. The corresponding regularity class for density $\rho$ follows from its connection to $u$ through the $e$-quantity which itself is of lower order. Hence, $\rho \in H^{2+\alpha}$. Indeed, it is proved in [14] for $1 \leqslant \alpha<2$ and in [5] for $0<\alpha<1$, that for any initial condition $\left(\rho_{0}, u_{0}\right) \in H^{2+\alpha} \times H^{3}$ away from vacuum there exists a unique local solution in the same class $(\rho, u) \in L^{\infty}\left([0, T) ; H^{2+\alpha} \times H^{3}\right)$. We note that since the argument [14] for $1 \leqslant \alpha<2$ is not using the dissipative structure of the commutator term, it can be easily adapted to the case $0<\alpha<1$. Both results [14] and [5] are accompanied by a BKM type continuation criterion which enables to extend the solution beyond any finite $T$. 
- (Pointwise bound on the density). We have the pointwise lower- and upper-bound on the density globally on the interval of existence

$$
0<c_{0} \leqslant \rho(x, t) \leqslant C_{0}, \quad x \in \mathbb{T}, t \geqslant 0,
$$

where the constants $c_{0}$ and $C_{0}$ depend only on the initial condition. This was established in [15] following a weaker lower bound $\rho \gtrsim 1 /(1+t)$ found in [14, 5].

- (Strong alignment). The variation of the velocity, $\max _{y} u(y, t)-\min _{y} u(y, t)$, is contracting exponentially fast,

$$
\frac{\mathrm{d}}{\mathrm{d} t} V(t) \leqslant-c_{1} V(t), \quad V(t):=\max _{y} u(y, t)-\min _{y} u(y, t),
$$

hence there is an exponentially fast alignment of velocities to their average value $u(x, t) \rightarrow$ $\bar{u}=\mathcal{P}_{0} / \mathcal{M}_{0}$.

- (Fractional parabolic enhancement). The parabolic nature of both the momentum and mass equations is an essential structural feature of the system that has been used in all of the preceding works. Using the e-quantity we can write

$$
\rho_{t}+u \rho_{x}+e \rho=-\rho \Lambda^{\alpha} \rho .
$$

The drift $u$ and the forcing $e \rho$ are bounded a priori due to the maximum principle stated above. Moreover, utilizing the boundedness of $\rho$ and of $e=u_{x}-\Lambda^{\alpha} \rho$ we immediately conclude for $0<\alpha<1$ that $u(\cdot, t) \in C^{1-\alpha}$ uniformly in time. Hence, the mass equation falls under the general class of fractional parabolic equations,

$$
w_{t}+b \cdot \nabla_{x} w=\mathcal{L}_{\alpha} w+f, \quad \mathcal{L}_{\alpha} w(x)=\int_{\mathbb{R}} K(x, z, t)(w(x+z)-w(x)) \mathrm{d} z
$$

with a diffusion operator associated with the singular kernel $K(x, z, t)=\rho(x+z)|z|^{-(1+\alpha)}$, and $f \in L^{\infty}, b \in C^{1-\alpha}$. Regularity of these equations has been the subject of active research in recent years. In particular, the result of Silvestre [16], see also Schwab and Silverstre [13], states that there exists a $\gamma>0$ such that for all $t>0$,

$$
|\rho|_{C^{\gamma}(\mathbb{T} \times[1,2))} \lesssim|\rho|_{L^{\infty}(0,2)}+|\rho e|_{L^{\infty}(0,2)} .
$$

Since the right hand side is uniformly bounded on the entire line we have obtained uniform bounds on $C^{\gamma}$-norm starting, by rescaling, from any positive time.

\section{Proof of the MAIN RESUlT}

3.1. Existence of global smooth solutions. We begin with proving a uniform bound $\left|\rho_{x}(\cdot, t)\right|_{\infty}<\infty$. In particular, we then have a uniform bound on $\left|\Lambda^{\alpha} \rho\right|_{\infty}, e$, and hence on $\left|u^{\prime}\right|_{\infty}$ and this readily implies global existence by the BKM criterion $\int_{0}^{T}\left|u_{x}(\cdot, t)\right| \mathrm{d} x<\infty$. To simplify notations, we now use $\{\cdot\}^{\prime},\{\cdot\}^{\prime \prime}$ and so on to denote spatial differentiation.

Lemma 3.1. Under the assumptions stated of Theorem 1.1 the following uniform bound holds

$$
\sup _{t \geqslant 0}\left|\rho^{\prime}(\cdot, t)\right|_{\infty}<\infty
$$


Proof. Taking the derivative of the density equation we obtain

$$
\partial_{t} \rho^{\prime}+u \rho^{\prime \prime}+u^{\prime} \rho^{\prime}+e^{\prime} \rho+e \rho^{\prime}=-\rho^{\prime} \Lambda^{\alpha} \rho-\rho \Lambda^{\alpha} \rho^{\prime},
$$

and expressing, $u^{\prime}=e+\Lambda^{\alpha} \rho$, we rewrite the $\rho^{\prime}$-equation as

$$
\partial_{t} \rho^{\prime}+u \rho^{\prime \prime}+e^{\prime} \rho+2 e \rho^{\prime}=-2 \rho^{\prime} \Lambda^{\alpha} \rho-\rho \Lambda^{\alpha} \rho^{\prime} .
$$

Multiplying by $\rho^{\prime}$ and evaluating the equation at the point $x_{+}$which maximize $\left|\rho^{\prime}\left(x_{+}, t\right)\right|=$ $\max _{x}\left|\rho^{\prime}(x, t)\right|$ we obtain

$$
\frac{1}{2} \partial_{t}\left|\rho_{+}^{\prime}\right|^{2}+e_{+}^{\prime} \rho_{+} \rho_{+}^{\prime}+2 e_{+}\left|\rho_{+}^{\prime}\right|^{2}=-2\left|\rho_{+}^{\prime}\right|^{2} \Lambda^{\alpha} \rho_{+}-\rho_{+} \rho_{+}^{\prime} \Lambda^{\alpha} \rho_{+}^{\prime}=:-2\left|\rho^{\prime}\right|^{2} \cdot I+I I .
$$

In view of (2.7) and (2.5) the whole nonlinear term on the left hand side can be estimated by

$$
\left.\left.\left|e_{+}^{\prime} \rho_{+} \rho_{+}^{\prime}+2 e_{+}\right| \rho_{+}^{\prime}\right|^{2}\left|\leqslant c_{2}\right| \rho_{+}^{\prime}\right|^{2}
$$

Next, in view of the lower-bound $\rho \geqslant c_{0}$, we have

$$
I I=\rho_{+} \rho_{+}^{\prime} \Lambda^{\alpha} \rho_{+}^{\prime} \geqslant \frac{1}{2} c_{0} \mathrm{D}_{\alpha} \rho^{\prime}\left(x_{+}\right),
$$

where

$$
\mathrm{D}_{\alpha} \rho^{\prime}(x):=\int_{\mathbb{R}} \frac{\left|\rho^{\prime}(x)-\rho^{\prime}(x+z)\right|^{2}}{|z|^{1+\alpha}} \mathrm{d} z .
$$

By the nonlinear maximum principle of [3], at the maximal point $x=x_{+}$we have

$$
\mathrm{D}_{\alpha} \rho^{\prime}\left(x_{+}\right) \geqslant c_{3} \frac{\left|\rho_{+}^{\prime}\right|^{2+\alpha}}{|\rho|_{\infty}} \geqslant c_{4}\left|\rho_{+}^{\prime}\right|^{2+\alpha}
$$

and hence

$$
I I=-\rho_{+} \rho_{+}^{\prime} \Lambda^{\alpha} \rho_{+}^{\prime} \leqslant-c_{5}\left|\rho_{+}^{\prime}\right|_{\infty}^{2+\alpha}, \quad c_{5}=\frac{1}{2} c_{0} c_{4} .
$$

We now get back to estimating the term $I=\Lambda^{\alpha} \rho$ in (3.2). The estimates are not restricted to the maximal point $x_{+}$so we temporarily drop the subscript $\{\cdot\}_{+}$. Let $\psi \in C^{\infty}$ be the usual even cut-off function with $\psi(z)=1$ for $|z|<1$ and $\psi(z)=0$ for $|z|>2$. Denote $\psi_{r}(z)=\psi(z / r)$, and decompose

$$
\begin{aligned}
\Lambda^{\alpha} \rho(x)= & \int \psi_{r}(z) \frac{\rho(x)-\rho(x+z)}{|z|^{1+\alpha}} \mathrm{d} z+\int_{|z|<2 \pi}\left(1-\psi_{r}(z)\right) \frac{\rho(x)-\rho(x+z)}{|z|^{1+\alpha}} \mathrm{d} z \\
& +\int_{2 \pi<|z|}\left(1-\psi_{r}(z)\right) \frac{\rho(x)-\rho(x+z)}{|z|^{1+\alpha}} \mathrm{d} z=: I_{1}+I_{2}+I_{3} .
\end{aligned}
$$

The last integral, $I_{3}$, is bounded by a constant multiple of $|\rho|_{\infty}$, which is uniformly bounded, $\leqslant c_{6}$. In the intermediate integral we use $C^{\gamma}$-regularity of $\rho$ and the fact that the region of integration is restricted to $|z|>r$. So, we obtain

$$
I_{2}=\left|\int_{|z|<2 \pi}\left(1-\psi_{r}(z)\right) \frac{\rho(x)-\rho(x+z)}{|z|^{1+\alpha}} \mathrm{d} z\right| \leqslant c_{7} r^{\gamma-\alpha} .
$$

For the first small-scale integral, we use that $|z|^{-1-\alpha}=-\frac{1}{\alpha} \partial_{z}\left(z|z|^{-1-\alpha}\right)$ and integrate by parts to obtain

$$
I_{1}=\int \psi_{r}(z) \frac{\rho(x)-\rho(x+z)}{|z|^{1+\alpha}} \mathrm{d} z=\frac{1}{\alpha} \int \psi_{r}^{\prime}(z) \frac{\rho(x)-\rho(x+z)}{|z|^{1+\alpha}} z \mathrm{~d} z-\frac{1}{\alpha} \int \psi_{r}(z) \frac{\rho^{\prime}(x+z)}{|z|^{1+\alpha}} z \mathrm{~d} z .
$$


In the first integral we use $C^{\gamma}$ regularity to obtain an upper-bound $\lesssim r^{\gamma-\alpha}$; as to the second, since $\psi_{r}$ is even we can add the term $\rho^{\prime}(x)$ inside,

$$
\frac{1}{\alpha} \int \psi_{r}(z) \frac{\rho^{\prime}(x+z)}{|z|^{1+\alpha}} z d z=\frac{1}{\alpha} \int \psi_{r}(z) \frac{\rho^{\prime}(x+z)-\rho^{\prime}(x)}{|z|^{1+\alpha}} z \mathrm{~d} z,
$$

and using Hölder, the last integral does not exceed $\leqslant c_{8}\left(\mathrm{D}_{\alpha} \rho^{\prime}\right)^{1 / 2}(x) r^{1-\alpha / 2}$. Putting all these estimates of $I_{1}, I_{2}$ and $I_{3}$ together, we obtain the bound for the nonlinear term $-2\left|\rho^{\prime}\right|^{2} I$,

$$
\begin{aligned}
\left.|| \rho_{+}^{\prime}\right|^{2} \Lambda^{\alpha} \rho_{+} \mid & \lesssim c_{6}\left|\rho_{+}^{\prime}\right|^{2}+c_{7}\left|\rho_{+}^{\prime}\right|^{2} r^{\gamma-\alpha}+c_{8}\left|\rho_{+}^{\prime}\right|^{2}\left(\mathrm{D}_{\alpha} \rho_{+}^{\prime}\right)^{1 / 2}(x) r^{1-\alpha / 2} \\
& \leqslant c_{6}\left|\rho_{+}^{\prime}\right|^{2}+c_{7}\left|\rho_{+}^{\prime}\right|^{2} r^{\gamma-\alpha}+\frac{c_{0}}{4} \mathrm{D}_{\alpha} \rho^{\prime}\left(x_{+}\right)+c_{9} r^{2-\alpha}\left|\rho^{\prime}\right|^{4} .
\end{aligned}
$$

The third term on the right, $\frac{c_{0}}{4} \mathrm{D}_{\alpha} \rho^{\prime}\left(x_{+}\right)$is absorbed into (3.3), leaving us with the dissipation of $\frac{1}{2} I I \leqslant-\frac{c_{5}}{2}\left|\rho_{+}^{\prime}\right|^{2+\alpha}$ in (3.4). Setting $r=\frac{c_{10}}{\left|\rho_{+}^{\prime}\right|}$ with sufficiently small $c_{10}$, we see that the second and fourth terms on the right hand side of (3.5) are absorbed into the dissipation term $\frac{1}{2} I I$. With such choice of $r$, the final bound of (3.2) reads,

$$
\partial_{t}\left|\rho_{+}^{\prime}\right|^{2} \leqslant c_{11}\left|\rho_{+}^{\prime}\right|^{2}+c_{12}\left|\rho_{+}^{\prime}\right|^{2+\alpha-\gamma}-c_{13}\left|\rho_{+}^{\prime}\right|^{2+\alpha},
$$

which implies the claimed control of $\left|\rho^{\prime}(\cdot, t)\right|_{\infty}$.

3.2. Main theorem - step 1: exponential decay towards a flocking state. To establish the stated exponential decay of $\left|u_{x}(\cdot, t)\right|$ we first prepare with the following refinement of the nonlinear maximum principle, [3] extending [15, Lemma 3.3].

Lemma 3.2 (Enhancement of dissipation by small amplitudes). Let $u \in C^{1}(\mathbb{T})$ be a given function with amplitude $V=\max u-\min u$. There is an absolute constant $c_{1}>0$ such that the following pointwise estimate holds

$$
D_{\alpha} u^{\prime}(x)=\int_{\mathbb{R}} \frac{\left|u^{\prime}(x)-u^{\prime}(x+z)\right|^{2}}{|z|^{1+\alpha}} d z \geqslant c_{1} \frac{\left|u^{\prime}(x)\right|^{2+\alpha}}{V^{\alpha}}, \quad V=\max u-\min u .
$$

In addition, there is an absolute constant $c_{2}>0$ such that for all $B>0$ one has

$$
D_{\alpha} u^{\prime}(x) \geqslant B\left|u^{\prime}(x)\right|^{2}-c_{2} B^{\frac{1+\alpha}{\alpha}} V^{2} .
$$

Proof. Let $\psi_{r}$ be as in the proof of Lemma 3.1. Discarding the positive term $|u(x+z)|^{2}$ we obtain

$$
\begin{aligned}
\mathrm{D}_{\alpha} u^{\prime}(x) & \geqslant \int_{|z|>r}\left(1-\psi_{r}(z)\right) \frac{\left|u^{\prime}(x)\right|^{2}-2 u^{\prime}(x+z) u^{\prime}(x)}{|z|^{1+\alpha}} \mathrm{d} z \\
& =c_{1}\left|u^{\prime}(x)\right|^{2} r^{-\alpha}-2 u^{\prime}(x) \int_{|z|>r}\left(1-\psi_{r}(z)\right) \frac{u^{\prime}(x+z)}{|z|^{1+\alpha}} \mathrm{d} z .
\end{aligned}
$$

Now, using $u^{\prime}(x+z) \equiv(u(x+z)-u(x))_{z}$ we integrate by parts in the second integral to obtain

$$
\begin{aligned}
& \int_{|z|>r}\left(1-\psi_{r}(z)\right) \frac{u^{\prime}(x+z)}{|z|^{1+\alpha}} \mathrm{d} z \\
& \quad=\int_{r<|z|<2 r} \psi_{r}^{\prime}(z) \frac{u(x+z)-u(x)}{|z|^{1+\alpha}} \mathrm{d} z+(1+\alpha) \int_{|z|>r}\left(1-\psi_{r}(z)\right) \frac{u(x+z)-u(x)}{|z|^{3+\alpha}} z \mathrm{~d} z .
\end{aligned}
$$


Both integrals are bounded by a constant multiple of $V r^{-(1+\alpha)}$. Hence

$$
\mathrm{D}_{\alpha} u^{\prime}(x) \geqslant c_{1}\left|u^{\prime}(x)\right|^{2} r^{-\alpha}-c_{2}\left|u^{\prime}(x)\right| V r^{-(1+\alpha)} .
$$

Picking $r=\frac{2 c_{2} V}{c_{1}\left|u^{\prime}(x)\right|}$ we obtain (3.7). Picking $r=B^{-(1 / \alpha)}$ and using Young's inequality,

$$
\mathrm{D} u^{\prime}(x) \geqslant c_{1} B\left|u^{\prime}(x)\right|^{2}-c_{2}\left|u^{\prime}(x)\right| V B^{\frac{1+\alpha}{\alpha}} \geqslant c_{3} B\left|u^{\prime}(x)\right|^{2}-c_{4} B^{\frac{2+\alpha}{\alpha}} V^{2},
$$

we obtain (3.8).

Lemma 3.3. Under the assumptions of Theorem 1.1 there exist constants $C, \delta>0$ such that for all $t>0$ one has

$$
\left|u^{\prime}(\cdot, t)\right|_{\infty} \leqslant C e^{-\delta t}
$$

Proof. Differentiating the $u$-equation and evaluating at a point of maximum we find

$$
\frac{\mathrm{d}}{\mathrm{d} t}\left|u^{\prime}\right|^{2} \leqslant\left|u^{\prime}\right|^{3}+\mathcal{T}\left(\rho^{\prime}, u\right) u^{\prime}+\mathcal{T}\left(\rho, u^{\prime}\right) u^{\prime}, \quad \mathcal{T}(\rho, u):=-\Lambda^{\alpha}(\rho u)+u \Lambda^{\alpha}(\rho) .
$$

Pertaining to the dissipation term, let us observe

$$
\left(u^{\prime}(y)-u^{\prime}(x)\right) u^{\prime}(x)=-\frac{1}{2}\left|u^{\prime}(y)-u^{\prime}(x)\right|^{2}+\frac{1}{2}\left(\left|u^{\prime}(y)\right|^{2}-\left|u^{\prime}(x)\right|^{2}\right) \leqslant-\frac{1}{2}\left|u^{\prime}(y)-u^{\prime}(x)\right|^{2} .
$$

Thus, in view of density bounds (2.7),

$$
\mathcal{T}\left(\rho, u^{\prime}\right) u^{\prime}(x) \leqslant-c_{1} \mathrm{D}_{\alpha} u^{\prime}(x) .
$$

The dissipation encoded in $-c_{1} \mathrm{D}_{\alpha} u^{\prime}(x)$ cannot control the full cubic term $\left|u^{\prime}\right|^{3}$ on the right of (3.10); yet as noted earlier, the term $\left|u^{\prime}\right|$ is uniformly bounded (by the bounds of $\left|\Lambda^{\alpha} \rho\right|_{\infty}$ and $\left.|e|_{\infty}\right)$ and in view of the enhancement Lemma 3.2,

$$
\left|u^{\prime}\right|^{3} \lesssim\left|u^{\prime}\right|^{2+\alpha} \lesssim V^{\alpha}(t) D_{\alpha} u^{\prime}, \quad V(t)=\max _{y} u(y, t)-\min _{y} u(y, t) .
$$

Thus, the latter bound on $\left|u^{\prime}\right|^{3}$ can be absorbed into dissipation term, at least after a finite time at which $V(t)$ becomes small enough below certain threshold, $V(t)<c_{1}$.

Let us turn to the remaining term $\mathcal{T}\left(\rho^{\prime}, u\right) u^{\prime}$. We have

$$
\begin{aligned}
\left|\mathcal{T}\left(\rho^{\prime}, u\right) u^{\prime}\right|= & \left|u^{\prime}\right| \int_{|z|<2 \pi}\left|\rho^{\prime}(x+z)\right| \frac{|u(x+z)-u(x)|}{|z|^{1+\alpha}} \mathrm{d} z \\
& +\left|u^{\prime}\right| \int_{|z|>2 \pi}\left|\rho^{\prime}(x+z)\right| \frac{|u(x+z)-u(x)|}{|z|^{1+\alpha}} \mathrm{d} z \\
\leqslant & \left|u^{\prime}\right|_{\infty}^{2}\left|\rho^{\prime}\right|_{\infty}+\left|u^{\prime}\right|_{\infty}\left|\rho^{\prime}\right|_{\infty} V \leqslant c_{2}\left|u^{\prime}\right|_{\infty}^{2}+E,
\end{aligned}
$$

where $E$ denotes a generic exponentially decaying quantity. In view of (3.8), the quadratic term gets absorbed into dissipation leaving only exponentially decaying source term:

$$
\frac{\mathrm{d}}{\mathrm{d} t}\left|u^{\prime}\right|^{2} \leqslant E-c_{3}\left|u^{\prime}\right|^{2}
$$

for all $t>t_{0}$ for some large $t_{0}$. The result follows by integration.

We are now ready to prove existence of a flocking pair, at this stage in rough spaces. 
Lemma 3.4. Under the assumptions of Theorem 1.1 there exist $C, \delta>0$ and a flocking pair $(\bar{u}, \bar{\rho}) \in \mathcal{F}, \bar{\rho} \in C^{1-\varepsilon}$, for every $\varepsilon>0$, such that

$$
|\rho(\cdot, t)-\bar{\rho}(\cdot, t)|_{\infty} \leqslant C e^{-\delta t}, \quad t>0 .
$$

Thus, $\mathcal{F}$ contains all limiting states of the system (1.1).

Proof. The proof is identical to one given in [15]. We include it for completeness. Clearly, the velocity goes to its natural limit $\bar{u}=\mathcal{P}_{0} / \mathcal{M}_{0}$. We pass to the moving reference frame and denote $\widetilde{\rho}(x, t):=\rho(x+t \bar{u}, t)$. We see that $\widetilde{\rho}$ satisfies

$$
\widetilde{\rho}_{t}+(u-\bar{u}) \widetilde{\rho}_{x}+u_{x} \widetilde{\rho}=0,
$$

where all the $u$ 's are evaluated at $x+t \bar{u}$. According to the established bounds we have $\left|\widetilde{\rho}_{t}\right|_{\infty}<C e^{-\delta t}$. This proves that $\widetilde{\rho}(\cdot, t)$ is Cauchy as $t \rightarrow \infty$, and hence there exists a unique limiting state, $\rho_{\infty}(x)$, such that

$$
\left|\widetilde{\rho}(\cdot, t)-\rho_{\infty}(\cdot)\right|_{\infty}<C_{1} e^{-\delta t}
$$

Denoting $\bar{\rho}(\cdot, t)=\rho_{\infty}(x-t \bar{u})$ completes the proof of (3.11). The membership of $\bar{\rho}$ in $C^{1-\varepsilon}$ follows from Lemma 3.1 and the compactness.

3.3. Main theorem - step 2: decay of higher derivatives. We start by showing exponential decay of $\left|u^{\prime \prime}\right|_{\infty}$. As before we denote by $E=E(t)$ any quantity with an exponential decay. For example, at this point we know that $\left|u^{\prime}\right|_{\infty}=E$ and $V=E$. According to Lemma 3.2 applied to $u^{\prime \prime}$, we have the following pointwise bounds

$$
\begin{aligned}
& \mathrm{D}_{\alpha} u^{\prime \prime}(x) \geqslant \frac{\left|u^{\prime \prime}(x)\right|^{2+\alpha}}{E} \\
& \mathrm{D}_{\alpha} u^{\prime \prime}(x) \geqslant B\left|u^{\prime \prime}(x)\right|^{2}-C(B) E .
\end{aligned}
$$

Due to these bounds the dissipation term absorbs all $(2+\alpha)$-power terms $C\left|u^{\prime \prime}\right|^{2+\alpha}$ as well as quadratic terms with bounded coefficients $C\left|u^{\prime \prime}\right|^{2}$, and by Young any linear term $E\left|u^{\prime \prime}\right|$ with exponentially decaying coefficient. The cost of this absorbing is a free source term of type $E$.

Lemma 3.5. Under the assumptions of Theorem 1.1, there are constants $C, \delta>0$ such that for all $t>0$ one has

$$
\left|u^{\prime \prime}(\cdot, t)\right|_{\infty} \leqslant C e^{-\delta t}
$$

Proof. Evaluating the $u^{\prime \prime}$-equation at a point of maximum and performing the same initial steps as in Lemma 3.3 we obtain

$$
\frac{\mathrm{d}}{\mathrm{d} t}\left|u^{\prime \prime}\right|^{2} \leqslant E\left|u^{\prime \prime}\right|^{2}-c_{0} \mathrm{D}_{\alpha} u^{\prime \prime}(x)+\mathcal{T}\left(\rho^{\prime \prime}, u\right) u^{\prime \prime}+2 \mathcal{T}\left(\rho^{\prime}, u^{\prime}\right) u^{\prime \prime} .
$$

As elaborated above, the quadratic term can be absorbed into dissipation by cost of an exponentially decaying source:

$$
\frac{\mathrm{d}}{\mathrm{d} t}\left|u^{\prime \prime}\right|^{2} \leqslant E-c_{1} \mathrm{D}_{\alpha} u^{\prime \prime}(x)+\mathcal{T}\left(\rho^{\prime \prime}, u\right) u^{\prime \prime}+2 \mathcal{T}\left(\rho^{\prime}, u^{\prime}\right) u^{\prime \prime} .
$$

We now focus on $\mathcal{T}\left(\rho^{\prime \prime}, u\right) u^{\prime \prime}$. Unfortunately, at this point we do not have any uniform control on $\left|\rho^{\prime \prime}\right|$. Thus, we will need to move one or $1-\alpha$ derivative from $\rho^{\prime \prime}$. To achieve this we add 
and subtract $z u^{\prime}(x)$ inside the integral. We obtain

$$
\begin{aligned}
\mathcal{T}\left(\rho^{\prime \prime}, u\right) u^{\prime \prime}= & u^{\prime \prime}(x) u^{\prime}(x) \int \rho^{\prime \prime}(x+z) \frac{z}{|z|^{1+\alpha}} \mathrm{d} z \\
& +u^{\prime \prime}(x) \int \rho^{\prime \prime}(x+z)\left(u(x+z)-u(x)-z u^{\prime}(x)\right) \frac{\mathrm{d} z}{|z|^{1+\alpha}} \\
& =: u^{\prime \prime}(x) u^{\prime}(x) \cdot I+u^{\prime \prime}(x) \cdot I I .
\end{aligned}
$$

We now integrate by parts both integrals, $I$ and $I I$. In the first we obtain

$$
\begin{aligned}
I=\int \rho^{\prime \prime}(x+z) \frac{z}{|z|^{1+\alpha}} \mathrm{d} z & =\int\left(\rho^{\prime}(x+z)-\rho^{\prime}(x)\right) \frac{z}{|z|^{1+\alpha}} \mathrm{d} z \\
& =\alpha \int\left(\rho^{\prime}(x+z)-\rho^{\prime}(x)\right) \frac{\mathrm{d} z}{|z|^{1+\alpha}}=-\alpha \Lambda^{\alpha} \rho^{\prime}(x) .
\end{aligned}
$$

Note that $\Lambda^{\alpha} \rho^{\prime}(x)=e^{\prime}-u^{\prime \prime}$, and $\left|e^{\prime}\right| \lesssim\left|\rho^{\prime}\right|<C$. Consequently,

$$
\left|u^{\prime \prime}(x) u^{\prime}(x) \cdot I\right|=\left|-\alpha \Lambda^{\alpha} \rho^{\prime}(x) u^{\prime \prime}(x) u^{\prime}(x)\right| \leqslant E\left|u^{\prime \prime}\right|^{2}+E\left|u^{\prime \prime}\right|,
$$

both are absorbed into dissipation with an extra $E$-term. In the second integral, we obtain

$$
\begin{aligned}
I I= & -\int \rho^{\prime}(x+z)\left(u^{\prime}(x+z)-u^{\prime}(x)\right) \frac{\mathrm{d} z}{|z|^{1+\alpha}} \\
& +c \int \rho^{\prime}(x+z)\left(u(x+z)-u(x)-z u^{\prime}(x)\right) \frac{z \mathrm{~d} z}{|z|^{3+\alpha}}
\end{aligned}
$$

Splitting each integral into $|z|<2 \pi$ and $|z|>2 \pi$ regions, and using Taylor in the small scale regions we immediately obtain the bound $\lesssim\left|\rho^{\prime}\right|\left|u^{\prime}\right|+\left|\rho^{\prime}\right|\left|u^{\prime \prime}\right| \leqslant E+c\left|u^{\prime \prime}\right|$. The corresponding term $u^{\prime \prime}(x) \cdot I I$ is therefore bounded by $E\left|u^{\prime \prime}\right|+c\left|u^{\prime \prime}\right|^{2}$, which is again absorbed into dissipation. We conclude that the whole term $\mathcal{T}\left(\rho^{\prime \prime}, u\right) u^{\prime \prime}$ is dominated by dissipative term plus an $E$ source.

It remains to notice that the $\mathcal{T}\left(\rho^{\prime}, u^{\prime}\right) u^{\prime \prime}$ term is precisely given by the first integral on the right hand side of (3.15), which has been estimated already. We arrive at

$$
\frac{\mathrm{d}}{\mathrm{d} t}\left|u^{\prime \prime}\right|^{2} \leqslant E-c \mathrm{D} u^{\prime \prime}(x) \lesssim E-\left|u^{\prime \prime}(x)\right|^{2} .
$$

This finishes the proof.

To proceed, let us note that we have automatically obtained the uniform bound

$$
\sup _{t}\left|\Lambda^{\alpha} \rho^{\prime}(\cdot, t)\right|_{\infty}<\infty .
$$

We are now in a position to perform final estimates in the top regularity class $H^{3} \times H^{2+\alpha}$.

Lemma 3.6. Under the assumptions of Theorem 1.1, there are constants $C, \delta>0$ such that for all $t>0$ one has

$$
\begin{aligned}
\left|u^{\prime \prime \prime}(\cdot, t)\right|_{2} & \leqslant C e^{-\delta t} \\
\left|\Lambda^{\alpha} \rho^{\prime \prime}(\cdot, t)\right|_{2} & \leqslant C .
\end{aligned}
$$

Proof of Lemma 3.6. Let us write the equation for $u^{\prime \prime \prime}$ :

$$
u_{t}^{\prime \prime \prime}+u u_{x}^{\prime \prime \prime}+4 u^{\prime} u^{\prime \prime \prime}+3 u^{\prime \prime} u^{\prime \prime}=\mathcal{T}\left(\rho^{\prime \prime \prime}, u\right)+3 \mathcal{T}\left(\rho^{\prime \prime}, u^{\prime}\right)+3 \mathcal{T}\left(\rho^{\prime}, u^{\prime \prime}\right)+\mathcal{T}\left(\rho, u^{\prime \prime \prime}\right) .
$$


Testing with $u^{\prime \prime \prime}$ we obtain (we suppress integral signs and note that $\int u^{\prime \prime} u^{\prime \prime} u^{\prime \prime \prime}=0$ )

$$
\begin{aligned}
\frac{\mathrm{d}}{\mathrm{d} t}\left|u^{\prime \prime \prime}\right|_{2}^{2} & \left.=-7 u^{\prime}\left(u^{\prime \prime \prime}\right)^{2}+2 \mathcal{T}\left(\rho^{\prime \prime \prime}, u\right) u^{\prime \prime \prime}+6 \mathcal{T}\left(\rho^{\prime \prime}, u^{\prime}\right) u^{\prime \prime \prime}+6 \mathcal{T}\left(\rho^{\prime}, u^{\prime \prime}\right)\right) u^{\prime \prime \prime}+2 \mathcal{T}\left(\rho, u^{\prime \prime \prime}\right) u^{\prime \prime \prime} \\
& \leqslant E\left|u^{\prime \prime \prime}\right|_{2}^{2}-c_{0} \int \mathrm{D}_{\alpha} u^{\prime \prime \prime} \mathrm{d} x+2 \mathcal{T}\left(\rho^{\prime \prime \prime}, u\right) u^{\prime \prime \prime}+6 \mathcal{T}\left(\rho^{\prime \prime}, u^{\prime}\right) u^{\prime \prime \prime}+6 \mathcal{T}\left(\rho^{\prime}, u^{\prime \prime}\right) u^{\prime \prime \prime}
\end{aligned}
$$

Note that $\int \mathrm{D}_{\alpha} u^{\prime \prime \prime} \mathrm{d} x=\left|u^{\prime \prime \prime}\right|_{H^{\alpha / 2}}^{2}$. From Lemma 3.2 we have the lower bound

$$
\int_{\mathbb{T}} \mathrm{D} u^{\prime \prime \prime} \mathrm{d} x \geqslant B\left|u^{\prime \prime \prime}\right|_{2}^{2}-C(B) E, \text { for any } B>0 .
$$

Again, the dissipation absorbs all quadratic terms and linear terms with $E$-coefficient. Manipulations below are much similar to the ones we performed in the proof of Lemma 3.5 So, we proceed straight with computations. We have

$$
\begin{aligned}
\mathcal{T}\left(\rho^{\prime \prime \prime}, u\right) u^{\prime \prime \prime}= & \int_{\mathbb{T} \times \mathbb{R}} \frac{\rho^{\prime \prime \prime}(x+z)(u(x+z)-u(x)) u^{\prime \prime \prime}(x)}{|z|^{1+\alpha}} \mathrm{d} z \mathrm{~d} x \\
= & \int_{\mathbb{T} \times \mathbb{R}} \frac{\rho^{\prime \prime \prime}(x+z) z u^{\prime}(x) u^{\prime \prime \prime}(x)}{|z|^{1+\alpha}} \mathrm{d} z \mathrm{~d} x \\
& +\int_{\mathbb{T} \times \mathbb{R}} \frac{\rho^{\prime \prime \prime}(x+z)\left(u(x+z)-u(x)-z u^{\prime}(x)\right) u^{\prime \prime \prime}(x)}{|z|^{1+\alpha}} \mathrm{d} z \mathrm{~d} x \\
= & \alpha \int_{\mathbb{T}} \Lambda^{\alpha} \rho^{\prime \prime}(x) u^{\prime}(x) u^{\prime \prime \prime}(x) \mathrm{d} x+\int_{\mathbb{T} \times \mathbb{R}} \frac{\rho^{\prime \prime}(x+z)\left(u^{\prime}(x+z)-u^{\prime}(x)\right) u^{\prime \prime \prime}(x)}{|z|^{1+\alpha}} \mathrm{d} z \mathrm{~d} x \\
& +\int_{\mathbb{T} \times \mathbb{R}} \frac{\rho^{\prime \prime}(x+z)\left(u(x+z)-u(x)-z u^{\prime}(x)\right) u^{\prime \prime \prime}(x) z}{|z|^{3+\alpha}} \mathrm{d} z \mathrm{~d} x \\
\leqslant & \left|u^{\prime}\right|_{\infty}\left|\Lambda^{\alpha} \rho^{\prime \prime}\right|_{2}\left|u^{\prime \prime \prime}\right|_{2}+\left|\rho^{\prime \prime}\right|_{2}\left|u^{\prime \prime \prime}\right|_{2}\left|u^{\prime}\right|_{\infty}+\left|\rho^{\prime \prime}\right|_{2}\left|u^{\prime \prime \prime}\right|_{2}\left|u^{\prime \prime}\right|_{\infty} \leqslant E\left|\Lambda^{\alpha} \rho^{\prime \prime}\right|_{2}\left|u^{\prime \prime \prime}\right|_{2} \\
\leqslant & E\left|e^{\prime \prime}\right|_{2}^{2}+E\left|u^{\prime \prime \prime}\right|_{2}^{2},
\end{aligned}
$$

where the last term in absorbed into dissipation. Next,

$$
\mathcal{T}\left(\rho^{\prime \prime}, u^{\prime}\right) u^{\prime \prime \prime}=\int_{\mathbb{T} \times \mathbb{R}} \frac{\rho^{\prime \prime}(x+z)\left(u^{\prime}(x+z)-u^{\prime}(x)\right) u^{\prime \prime \prime}(x)}{|z|^{1+\alpha}} \mathrm{d} z \mathrm{~d} x,
$$

which is precisely an integral we already estimated below. Finally,

$$
\begin{aligned}
\mathcal{T}\left(\rho^{\prime}, u^{\prime \prime}\right) u^{\prime \prime \prime} & =\int_{\mathbb{T} \times \mathbb{R}} \frac{\rho^{\prime}(x+z)\left(u^{\prime \prime}(x+z)-u^{\prime \prime}(x)\right) u^{\prime \prime \prime}(x)}{|z|^{1+\alpha}} \mathrm{d} z \mathrm{~d} x \\
& =\int_{\mathbb{T}} \int_{|z|<2 \pi} \frac{\rho^{\prime}(x+z)\left(u^{\prime \prime}(x+z)-u^{\prime \prime}(x)\right) u^{\prime \prime \prime}(x)}{|z|^{1+\alpha}} \mathrm{d} z \mathrm{~d} x \\
& +\int_{\mathbb{T}} \int_{|z|>2 \pi} \frac{\rho^{\prime}(x+z)\left(u^{\prime \prime}(x+z)-u^{\prime \prime}(x)\right) u^{\prime \prime \prime}(x)}{|z|^{1+\alpha}} \mathrm{d} z \mathrm{~d} x \\
& \leqslant\left|\rho^{\prime}\right|_{\infty}\left|u^{\prime \prime \prime}\right|_{2}^{2}+\left|\rho^{\prime}\right|_{\infty}\left|u^{\prime \prime}\right|_{\infty}\left|u^{\prime \prime \prime}\right|_{2} \leqslant c\left|u^{\prime \prime \prime}\right|_{2}^{2}+E\left|u^{\prime \prime \prime}\right|_{2} .
\end{aligned}
$$

This term is entirely absorbed into dissipation. We have obtained the estimate

$$
\frac{\mathrm{d}}{\mathrm{d} t}\left|u^{\prime \prime \prime}\right|_{2}^{2} \leqslant E+E\left|e^{\prime \prime}\right|_{2}^{2}-\int \mathrm{D}_{\alpha} u^{\prime \prime \prime} \mathrm{d} x \text {. }
$$


It remains to close with a bound on $e^{\prime \prime}$ :

$$
\begin{aligned}
\frac{\mathrm{d}}{\mathrm{d} t}\left|e^{\prime \prime}\right|_{2}^{2} & \leqslant 3 u^{\prime} e^{\prime \prime} e^{\prime \prime}+2 u^{\prime \prime} e^{\prime} e^{\prime \prime}+u^{\prime \prime \prime} e e^{\prime \prime} \leqslant E\left|e^{\prime \prime}\right|_{2}^{2}+E\left|e^{\prime \prime}\right|_{2}+\left|u^{\prime \prime \prime}\right|^{2}\left|e^{\prime \prime}\right|^{2} \\
& \leqslant E\left|e^{\prime \prime}\right|_{2}^{2}+\frac{E}{\delta}+\delta\left|e^{\prime \prime}\right|_{2}^{2}+\frac{1}{\delta}\left|u^{\prime \prime \prime}\right|_{2}^{2}+\delta\left|e^{\prime \prime}\right|_{2}^{2},
\end{aligned}
$$

for every $\delta>0$. Combining with (3.21) and absorbing all quadratic terms of $\left|u^{\prime \prime \prime}\right|_{2}$ we obtain for $X=\left|u^{\prime \prime \prime}\right|_{2}^{2}+\left|e^{\prime \prime}\right|_{2}^{2}$ :

$$
\frac{\mathrm{d}}{\mathrm{d} t} X \leqslant \frac{E}{\delta}+c \delta X
$$

This implies that the exponential growth rate of $X$ is at most $c \delta$, which can be made arbitrarily small. In particular this implies that $\left|e^{\prime \prime}\right|_{2}$ has arbitrarily small exponential rate. Going back to (3.21) we find that $E\left|e^{\prime \prime}\right|_{2}^{2}$ is another $E$-type term, since the $E$ coefficient has a finite negative decay rate. Consequently, we obtain

$$
\frac{\mathrm{d}}{\mathrm{d} t}\left|u^{\prime \prime \prime}\right|_{2}^{2} \leqslant E-c\left|u^{\prime \prime \prime}\right|_{2}^{2}
$$

which proves the result for $\left|u^{\prime \prime \prime}\right|_{2}$. To finish the bound on density we go back to the $e^{\prime \prime}-$ equation with the obtained exponential decay of $u^{\prime \prime \prime}$ :

$$
\frac{\mathrm{d}}{\mathrm{d} t}\left|e^{\prime \prime}\right|_{2}^{2} \leqslant 3 u^{\prime} e^{\prime \prime} e^{\prime \prime}+2 u^{\prime \prime} e^{\prime} e^{\prime \prime}+u^{\prime \prime \prime} e e^{\prime \prime} \leqslant E\left(\left|e^{\prime \prime}\right|_{2}^{2}+\left|e^{\prime \prime}\right|_{2}\right)
$$

This readily implies global uniform bound on $\left|e^{\prime \prime}\right|_{2}$, and hence on $\left|\rho^{\prime \prime \prime}\right|_{2}$. This proves the lemma.

As a consequence we readily obtain the full statement of Theorem 1.1. In particular, the convergence for densities stated in (1.5) follows by interpolation between exponential decay in $L^{\infty}$ and uniform boundedness in $H^{2+\alpha}$. The fact that $\bar{\rho} \in H^{2+\alpha}$ is a simple consequence of uniform boundedness of $\rho(t)$ in $H^{2+\alpha}$ and weak compactness.

\section{REFERENCES}

[1] J. Carrillo, Y.-P. Choi, and S. Perez. A review on attractive-repulsive hydrodynamics for consensus in collective behavior. In N. Bellomo, P. Degond, and E. Tadmor, editors, Active Particles. Advances in Theory, Models, and Applications, volume 1. Birkhäuser, 2017.

[2] J. A. Carrillo, Y.-P. Choi, E. Tadmor, and C. Tan. Critical thresholds in 1D Euler equations with non-local forces. Math. Models Methods Appl. Sci., 26(1):185-206, 2016.

[3] P. Constantin and V. Vicol. Nonlinear maximum principles for dissipative linear nonlocal operators and applications. Geom. Funct. Anal., 22(5):1289-1321, 2012.

[4] F. Cucker and S. Smale. Emergent behavior in flocks. IEEE Trans. Automat. Control, 52(5):852-862, 2007.

[5] T. Do, A. Kiselev, L. Ryzhik, and C. Tan. Global regularity for the fractional euler alignment system. 2017. https://arxiv.org/abs/1701.05155.

[6] S. Y Ha and J. G Liu, A simple proof of the Cucker-Smale flocking dynamics and mean-field limit. Communications in Mathematical Sciences, 7(2), (2009) 297-325.

[7] S.-Y. Ha and E. Tadmor. From particle to kinetic and hydrodynamic descriptions of flocking. Kinet. Relat. Models, 1(3):415-435, 2008.

[8] A. Kiselev, F. Nazarov, and A. Volberg. Global well-posedness for the critical 2D dissipative quasigeostrophic equation. Invent. Math., 167(3):445-453, 2007.

[9] S. Motsch and E. Tadmor. A new model for self-organized dynamics and its flocking behavior. J. Stat. Phys., 144(5):923-947, 2011. 
[10] S. Motsch and E. Tadmor. Heterophilious dynamics enhances consensus. SIAM Rev., 56(4):577-621, 2014.

[11] J. Peszek. Discrete Cucker-Smale flocking model with a weakly singular weight. SIAM J. Math. Anal., 47(5), 3671-3686.

[12] D. Poyato and J. Soler. Euler-type equations and commutators in singular and hyperbolic limits of kinetic Cucker-Smale models, Math. Models Methods Appl. Sci. 27, 1089 (2017) 1089-1152.

[13] R. W. Schwab and L. Silvestre. Regularity for parabolic integro-differential equations with very irregular kernels. Anal. PDE, 9(3):727-772, 2016.

[14] R. Shvydkoy and E. Tadmor. Eulerian dynamics with a commutator forcing. Trans. Mathematics and Applications 1(1) (2017) 1-26.

[15] R. Shvydkoy and E. Tadmor. Eulerian dynamics with a commutator forcing ii: flocking. Discrete and Continuous Dynamical Systems-A, in press.

[16] L. Silvestre. Hölder estimates for advection fractional-diffusion equations. Ann. Sc. Norm. Super. Pisa Cl. Sci. (5), 11(4):843-855, 2012.

[17] E. Tadmor and C.Tan. Critical thresholds in flocking hydrodynamics with non-local alignment. Philos. Trans. R. Soc. Lond. Ser. A Math. Phys. Eng. Sci., 372(2028):20130401, 22, 2014.

Department of Mathematics, Statistics, and Computer Science, M/C 249, University of Illinois, ChicAgo, IL 60607, USA

E-mail address: shvydkoy@uic.edu

Department of Mathematics, Center for Scientific Computation and Mathematical ModEling (CSCAMM), And Institute for Physical Sciences \& Technology (IPST), University of Maryland, College Park

Current address: Institute for Theoretical Studies (ITS), ETH-Zurich, Clausiusstrasse 47, CH-8092 Zurich, SwitzerLand

E-mail address: tadmor@cscamm.umd.edu 